\title{
L1 (CD171) is highly expressed in gastrointestinal stromal tumors
}

\author{
Jussuf T Kaifi ${ }^{1}$, Andrea Strelow ${ }^{1}$, Paulus G Schurr ${ }^{1}$, Uta Reichelt ${ }^{2}$, Emre F Yekebas ${ }^{1}$, \\ Robin Wachowiak ${ }^{1}$, Alexander Quaas ${ }^{2}$, Tim Strate ${ }^{1}$, Hansjoerg Schaefer ${ }^{2}$, \\ Guido Sauter ${ }^{2}$, Melitta Schachner ${ }^{3}$ and Jakob R Izbicki ${ }^{1}$ \\ ${ }^{1}$ Department of General, Visceral and Thoracic Surgery, University Medical Center Hamburg-Eppendorf, \\ Hamburg, Germany; ${ }^{2}$ Institute for Pathology, University Medical Center Hamburg-Eppendorf, Hamburg, \\ Germany and ${ }^{3}$ Center for Molecular Neurobiology, University Medical Center Hamburg-Eppendorf, \\ Hamburg, Germany
}

\begin{abstract}
The treatment strategy for mesenchymal tumors of the gastrointestinal tract is based upon typing of the tumor. Especially differential diagnosis of gastrointestinal stromal tumors (GISTs) to leiomyomas is crucial for determining radicality of surgery. $L 1$ cell adhesion molecule (CD171) plays an essential role in tumor progression. The aim of this study was to determine expression of $L 1$ in GISTs, smooth muscle tumors, desmoid-type fibromatosis and peripheral nerve sheath tumors (PNSTs). We retrospectively analyzed a total of 129 surgically resected primary tumors or metastases of 72 GISTs, 29 smooth muscle tumors, seven PNSTs and 21 desmoid-type fibromatosis by immunohistochemistry for c-kit, CD34, smooth muscle actin, desmin, vimentin, S-100 and $L 1$ expression. $L 1$ expression was detected in $53(74 \%)$ of 72 GISTs but in none of 29 smooth muscle tumors or 21 desmoid-type fibromatosis $(P<0.01$ by Fisher's test). In all, four (57\%) of seven peripheral nerve sheath tumors were L1-positive. Survival analysis of 55 surgically completely resected GISTs presenting without metastasis at initial diagnosis revealed no tumor-specific death among $L 1$-negative patients $(P=0.13$ by log-rank test; median follow-up time 41 months) and one recurrence was observed $(P=0.12)$. Interestingly high levels of $L 1$ were seen in tumor vascular endothelial cells of smooth muscle tumors and PNSTs, but not in GISTs. Our data show that $L 1$ is highly expressed in GISTs but not in smooth muscle tumors and desmoid-type fibromatosis being important differential diagnoses. The trend towards a reduced survival of L1-positive patients in this study has to be further evaluated in future trials with higher patient numbers. Modern Pathology (2006) 19, 399-406. doi:10.1038/modpathol.3800547; published online 6 January 2006
\end{abstract}

Keywords: gastrointestinal stromal tumors; tumor markers; $L 1$ cell adhesion molecule

Gastrointestinal stromal tumors (GISTs) are a heterogeneous group of tumors originating from the interstitial cells of Cajal that are located in the nerve plexus of the muscularis in the gut wall. ${ }^{1}$ On primary manifestation the outcome of GISTs is hardly predictable and patients have a bad prognosis once relapse or metastasis is discovered. ${ }^{2,3}$ The remarkable antitumor activity of the protein kinase inhibitor Imatinib (Glivec ${ }^{\mathbb{R}}$ ) in GISTs requires accurate diagnosis of this neoplasia $v s$ other soft tissue tumors. ${ }^{4}$ Most GISTs occur in the stomach and small intestine, but rarely they also present in the esophagus and large intestine. ${ }^{5}$ Differential diagno-

Correspondence: Dr JT Kaifi, MD, Department of General, Visceral and Thoracic Surgery, University Medical Center HamburgEppendorf, Martinistrasse 52, 20246 Hamburg, Germany.

E-mail: jkaifi@uke.uni-hamburg.de

Received 16 September 2005; revised 10 November 2005; accepted 1 December 2005; published online 6 January 2006 sis is important since GISTs even with low mitotic rate have a higher risk of malignant behavior and therefore require more aggressive surgery in contrast to, for example, leiomyomas that need to be enucleated only. ${ }^{6,7}$ Several classifications of GISTs exist, one of them is the 'consensus approach of 2002' that considers primary tumor size and mitotic rate. ${ }^{1}$ The outcome clearly depends on these two parameters. ${ }^{8}$ Tyrosine kinase $c$-kit (CD117) and PDGFR play important roles for diagnosis and therapy of GISTs. ${ }^{1-4}$ Another immunopathological marker is CD34 that is expressed in $60-70 \%$ of cases. ${ }^{1-9}$ A definitive discrimination between benign and malignant GISTs, however, has not been achieved.

Cell adhesion molecule L1 (CD171), a 200$220 \mathrm{kDa}$ type I glycoprotein of the immunoglobulin superfamily, plays a role in development of the nervous system by regulating cell interactions, including neuronal migration. ${ }^{10,11}$ It also mediates 
neuron-neuron adhesion and neurite outgrowth on Schwann cells. ${ }^{11-13}$ L1 undergoes homophilic binding, but also heterophilic interactions, for example with integrins, have been described. ${ }^{14,15}$ L1 was detected on a variety of tumor cells of neuronal, mesothelial and epithelial origin, such as of neuroblastomas, melanomas, lymphomas, small cell lung, colon and breast carcinomas. ${ }^{16-22}$ After cleavage by a metalloproteinase (ADAM10) from the tumor cell surface $L 1$ can be detected in serum of cancer patients. ${ }^{10,23-26}$ Its presence in tumor and serum has a prognostic significance in ovarian, uterine and renal cell carcinomas and is associated with metastasis of melanomas. ${ }^{22,27,28}$

The aim of our study was to evaluate whether L1 could serve as a potential diagnostical marker and prognostic factor of GISTs compared to smooth muscle tumors, desmoid-type fibromatosis and PNSTs.

\section{Materials and methods}

\section{Patients and Samples}

This study was approved by the Ethics Committee of the Chamber of Physicians in Hamburg, Germany. Written informed consent was obtained from all patients for use of the resected samples. For this study, 72 patients with GIST, 29 with smooth muscle tumors, seven with PNSTs and 21 with desmoidtype fibromatosis that were surgically treated between 1985 and 2004 were chosen retrospectively. In 65 patients primary GISTs were examined, among these, additional four liver and two lymph node metastases occurred. In seven patients only liver metastases were available. 18 leiomyomas and 11 leiomyosarcoma (three primary tumors, five liver and two peritoneal metastases), seven PNSTs (five malignant (two primary tumors and three metastases from lung, liver and pancreas) and two benign) and 21 desmoid-type fibromatosis (seven abdominal and 14 extraabdominal) were also analyzed. Tumor samples from patients with mesenchymal tumors from the gastrointestinal tract that were possibly misdiagnosed at times when diagnostic criterias for GIST were not established, were recently reevaluated and re-classified retrospectively. Besides, histological criterias, the following antibodies were used for immunohistological (re-) classification of all tumors: CD117 (c-kit; rabbit polyclonal) (Dako, Glostrup, Denmark), CD34 (mouse IgG1) (Novocastra Laboratories Ltd, Newcastle, United Kingdom), desmin (clone DE-R-11) (Dako), muscle actin (mouse IgG1; clone HHF35) (Enzo Diagnostics Inc., NY, USA), and $S-100$ protein (polyclonal) (Dako). The proliferative index was determined with Ki-67 (MIB1; IgG1) (Dako) and was categorized as mitotic count of $<5 / 50$ high-power fields (HPF), 5-10/50 HPF and $>10 / 50$ HPF. All data including sex, histopathological data including tumor size, lymph node metastasis, tumor type and disease stage were obtained from the clinical and pathological records.
Immunohistochemical Staining of $L 1$ and Evaluation of Expression

Immunohistochemical staining for $L 1$ was performed on $5 \mu \mathrm{m}$ thick sections placed on precoated slides with 3-triethoxysilylpropylamin (Merck, Darmstadt, Germany) of formalin-fixed tissues. After deparaffinization with Rotihistole (Merck) and rehydration in ethanol and TBS $(0.05 \mathrm{M} ; \mathrm{pH} 7.6)$ containing $0.1 \%$ Tween 20 (Sigma, Deisenhofen, Germany), tissue sections were pretreated for $30 \mathrm{~min}$ in $1 \%$ ammonium chloride $\left(\mathrm{NH}_{4} \mathrm{Cl}\right)$ in TBS, for $15 \mathrm{~min}$ in $0.05 \mathrm{M}$ glycine/TBS and afterwards boiled with ChemMate ${ }^{\mathbb{R}}$ Target Retrieval Solution (Dako) in a microwave oven according to the manufacturer's instructions. Staining was performed with the avidin-biotin-peroxidase method (HRP-AEC System, Cell and Tissue Staining Kit; R\&D Systems, Minneapolis, MN, USA). The primary antibody, a murine anti-human $L 1$ monoclonal antibody (IgG ; $_{1}$ Clone UJ127) (NeoMarkers, Fremont, CA, USA) binding to the extracellular domain of this molecule, was diluted at 1:50 in Antibody Diluent (Dako) and slides were incubated overnight in a humidified chamber at $4^{\circ} \mathrm{C}$. For each sample one slide, a control section, was incubated with irrelevant murine monoclonal $\operatorname{IgG}_{1}$ (MOPC21; Sigma) as a negative control to determine unspecific binding. All washing steps were done with TBS containing $0.1 \%$ Tween 20. Counterstaining was performed with Mayer's Haematoxylin solution (Merck) for $7 \mathrm{~min}$. Finally, slides were cover-slipped with aqueous mounting medium (Aquatex ${ }^{\mathbb{R}}$; Merck). Peripheral nerves being present in nearly all sections served as internal positive controls. Additionally, peripheral nerve sections were stained separately. Immunohistochemical analysis and scoring was performed by three independent investigators (JTK, AS and UR).

\section{Survival Data}

Clinical follow-up data were obtained by reviewing the hospital records, direct communication with the attending physicians and from the Cancer Registry of Hamburg. Tumor-specific survival was calculated from the date of surgical excision of the primary tumor to the date of death or last follow-up. Patients who died from causes other than GIST were censored at the time of death. Patients whose death was clearly documented as attributable to GIST considered to have died of that disease; other deaths were not considered to have been caused by GIST. Recurrence-free survival was calculated from the date of surgical excision to the date of recurrence. Peritoneal carcinosis was found in two cases and distant metastasis that was exclusively liver metastasis in 10 patients (no other recurrence, for instance lung metastasis or local relapse, was observed in any patient). Only patients with complete follow-up, whose primary tumors had tumor-free resection margins (R0) after surgical 
removal and no metastasis (Mo) at time of surgery were included for survival analysis. Patients that died within 3 months after surgery were excluded. Data with these criterias were available in 55 GIST patients. Two patients died within 3 months after surgery (pulmonary embolism and hemorrhagic shock due to bleeding out of a gastric GIST) and of one patient no follow-up data could be generated. A total of 14 patients were excluded from survival analysis because they either presented with metastatic disease (M1) at initial diagnosis or were not completely resectable (R1 or R2) at primary surgery.

\section{Statistical Analysis}

SPSS for Windows (Version 11.5.1) (SPSS Inc., Chicago, IL, USA) was used for statistical analysis. Survival curves were plotted using the KaplanMeier method and analyzed using the log-rank test. Cross table statistics were performed with Fisher's test. Association of $L 1$ expression and tumor size were statistically analyzed by unpaired $t$-test and mitotic count by $\chi^{2}$ test. Significance statements refer to $P$-values of two-tailed tests that were $<0.05$.

\section{Results}

\section{Characteristics of the Patients}

A total of 129 patients were included in our study. Out of these, 72 patients were suffering from GISTs, 29 from smooth muscle tumors (18 from leiomyomas and 11 from leiomyosarcoma), 21 from desmoidtype fibromatosis (seven abdominal and 14 extraabdominal) and seven from PNSTs (five malignant and two benign schwannomas). Patients' characteristics are listed in Table 1. Briefly, the median age of GIST patients was 61 years, of smooth muscle tumor patients 59 years, of desmoid-type fibromatosis patients 42 years and of PNST patients 43 years. All 72 GISTs were $c$-kit-positive (100\%), 64 (88.9\%) were CD34-positive, four $(5.6 \%)$ positive for $S-100$ protein, $22(30.6 \%)$ for smooth muscle actin and three $(4.2 \%)$ for desmin. Concerning 29 smooth muscle tumors, none expressed $c$-kit, CD34 or $S-100$, $27(93.1 \%)$ were positive for smooth muscle actin, and $24(82.8 \%)$ for desmin. The seven schwannomas examined were $S$-100-positive exclusively. All desmoid-type fibromatosis tumors expressed vimentin. Patients' characteristics including location of primary tumors are listed in Table 1.

\section{L1 Expression in GISTs, Smooth Muscle Tumors, Desmoid-Type Fibromatosis and PNSTs}

Of all 72 GIST samples included in this study, 53 (74\%) were L1-positive (Table 1). In contrast, none of 29 immunostained smooth muscle tumor and 21 desmoid-type fibromatosis samples expressed $L 1$. In all, four $(57 \%)$ of seven immunostained PNSTs were
Table 1 Clinicopathological characteristics of patients and levels of $L 1$ expression in GISTs, smooth muscle tumors and PNSTs

\begin{tabular}{|c|c|c|}
\hline Variable & $\begin{array}{c}\text { No. of } \\
\text { patients }\end{array}$ & $\begin{array}{l}\text { No. of L1-positive } \\
\text { tumors }(\mathrm{n}(\%))\end{array}$ \\
\hline Total & 129 & $57(44)$ \\
\hline \multicolumn{3}{|c|}{ Gastrointestinal stromal tumors (GISTs) } \\
\hline Total & 72 & $53(74)$ \\
\hline \multicolumn{3}{|c|}{ Localization of primary tumor } \\
\hline Esophagus & 2 & $2(100)$ \\
\hline Stomach & 50 & $43(86)$ \\
\hline Small intestine & 16 & $5(31)$ \\
\hline Colorectal & 3 & $3(100)$ \\
\hline Unknown & 1 & 0 \\
\hline \multicolumn{3}{|c|}{ Smooth muscle tumors } \\
\hline Total & 29 & 0 \\
\hline Leiomyomas & 18 & 0 \\
\hline Leiomyosarcomas & 11 & 0 \\
\hline \multicolumn{3}{|c|}{ Localization of primary tumor } \\
\hline Esophagus & 5 & 0 \\
\hline Stomach & 7 & 0 \\
\hline Small intestine & 2 & 0 \\
\hline Colorectal & 5 & 0 \\
\hline Other & 10 & 0 \\
\hline \multicolumn{3}{|c|}{ Desmoid-type fibromatosis } \\
\hline Total & 21 & 0 \\
\hline \multicolumn{3}{|c|}{ Localization of primary tumor } \\
\hline Abdominal & 7 & 0 \\
\hline Extraabdominal & 14 & 0 \\
\hline \multicolumn{3}{|c|}{ Peripheral nerve sheath tumors (PNSTs) } \\
\hline Total & 7 & $4(57)$ \\
\hline Benign PNSTs & 2 & $2(100)$ \\
\hline Malignant PNSTs & 5 & $2(40)$ \\
\hline \multicolumn{3}{|c|}{ Localization of primary tumors } \\
\hline Stomach & 1 & $1(100)$ \\
\hline Small intestine & 1 & 0 \\
\hline Colorectal & 2 & $2(100)$ \\
\hline Other & 4 & $1(25)$ \\
\hline
\end{tabular}

L1-positive. Figure 1 shows representative staining patterns for $L 1$ of a primary GIST and a liver metastasis and coexpression of L1, c-kit and CD34 in a gastric GIST. No differences in terms of positivity or negativity of L1 expression were detected between primary tumors and metastases in any case. For statistical analysis of a correlation between L1 expression in GISTs, smooth muscle tumors, desmoid-type fibromatosis or PNSTs a cross table was used and Fisher's test was applied showing a significant correlation between L1 expression and GISTs in comparison to L1 expression in smooth muscle tumors $(P<0.01)$ and desmoidtype fibromatosis tumors $(P<0.01)$, but not to PNSTs $(P=0.39)$ (Table 2). No correlation was determined between $L 1$ expression and tumor size $(P=0.24$ by $t$-test) or proliferative index (mitotic counts per HPF) of GIST $\left(P=0.12\right.$ by $\chi^{2}$ test). $L 1$ was also expressed on normal interstitial cells of Cajal but 
Gastric GIST: L1+

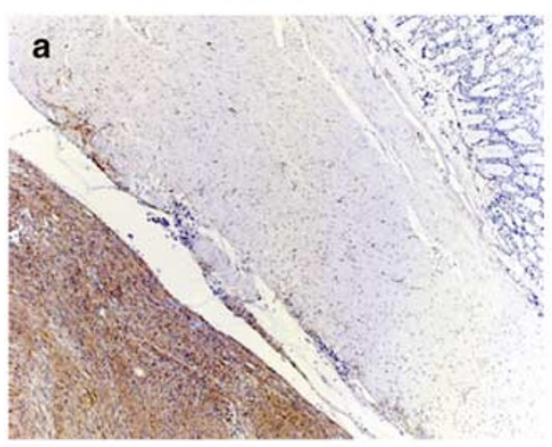

GIST: L1+

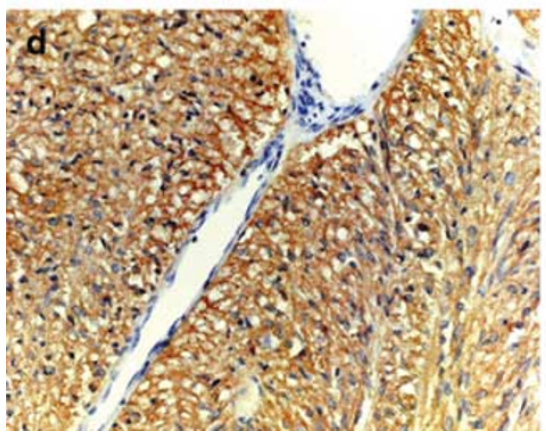

GIST metastasis: L1+

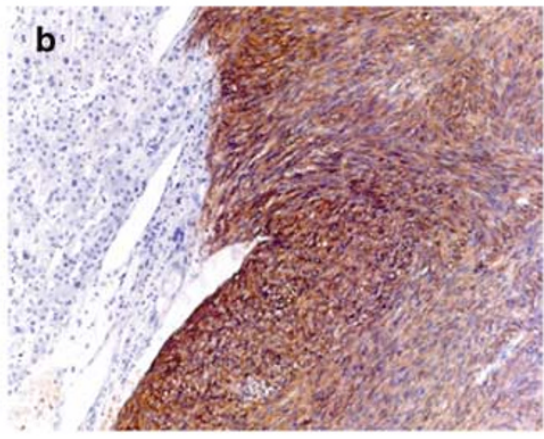

GIST: c-kit+

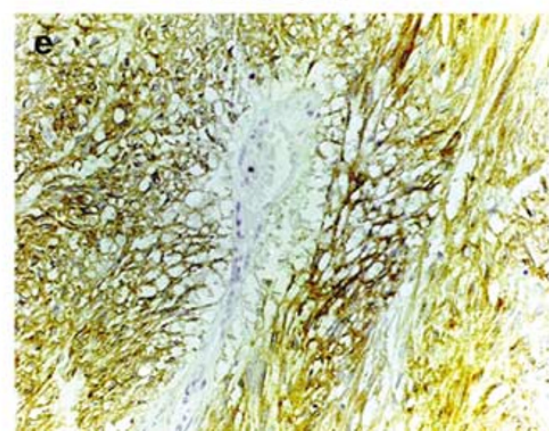

Gastric GIST: L1-

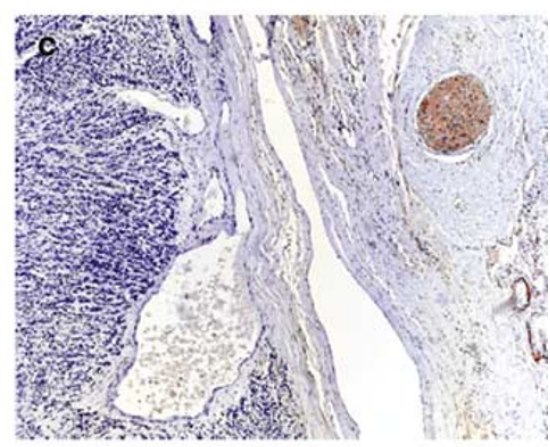

GIST: CD34+

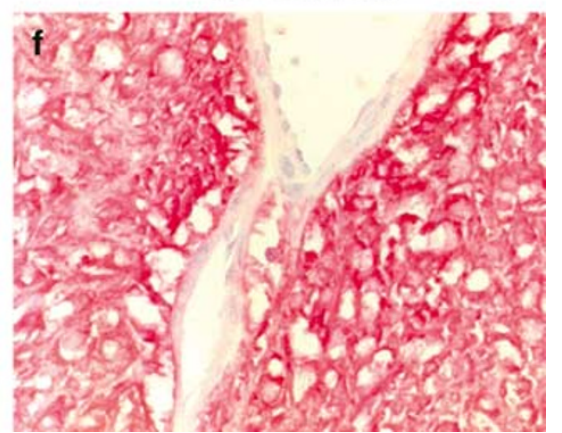

Figure 1 L1 expression in GISTs. Immunohistochemical staining was performed by the peroxidase method using monoclonal antibody against L1. (a) Represents an L1-positive gastric GIST, whereas (b) shows an L1-positive liver metastasis of a gastric GIST adjacent to normal liver tissue. (c) Shows an L1-negative gastric GIST and next to the tumor an L1-positive peripheral nerve. (d, e, f) Represent the same area of a gastric GIST positive for L1, c-kit and CD34, respectively (Magnification $\times 200$ ).

Table 2 L1 expression correlated between GISTs and smooth muscle tumors, desmoid-type fibromatosis or PNSTs

\begin{tabular}{lccr}
\hline & L1-negative & L1-positive & P-value \\
\hline GISTs & 19 & 53 & \\
Smooth muscle tumors & 29 & 0 & $<0.01$ \\
Desmoid-type fibromatosis & 21 & 0 & $<0.01$ \\
PNSTs & 3 & 4 & 0.39 \\
\hline
\end{tabular}

$P$-values were calculated by Fisher's test (two-sided) with GIST as reference entity.

not on the smooth muscular cells of the gastrointestinal tract. Interestingly, L1 was expressed in vascular endothelial cells of tumor vessels in smooth muscle tumors, but only in small and not in large vessels of PNSTs and in none of the GISTs examined (Figure 2).

\section{L1 Expression in GISTs and Survival}

Follow-up data of patients whose primary GIST was surgically completely resected (R0) and that did not present with distant metastasis (M0) at surgery were generated. Median follow-up time of all patients included for survival analysis $(n=55)$ was 41 months. The median follow-up time of survivors $(n=43)$ was 43 months, of L1-negative patients $(n=13) 43$ months and of L1-positive patients $(n=42) 41$ months. No tumor-specific death was observed in L1-negative patients $(n=13)$ in contrast to five death events among L1-positive patients $(n=42)$ (Figure 3). However, statistical analysis of the results with the log-rank test revealed that this difference did not reach the level of significance $(P=0.13)$. Analysis of recurrence-free survival revealed that one L1-negative patient developed recurrence in the form of liver metastasis 84 months after surgery (Figure 3). Among L1-positive patients, 11 events of recurrence where noted, nine developed liver metastasis and two peritoneal carcinosis. However, this difference was not significant ( $P=0.12$ by log-rank test). The patient with recurrence in the L1-negative arm is receiving Imatinib since 2002 and is still alive. In the L1-positive arm four patients are on medication with Imatinib since 2001 or 2002 and all are still living. Imatinib is applied to all patients on a daily basis at a dosage of $400 \mathrm{mg}$ orally.

\section{Discussion}

In an attempt to identify new markers for the differential diagnosis of GISTs from other soft tissue 
GIST: L1+ (vessel L1-)

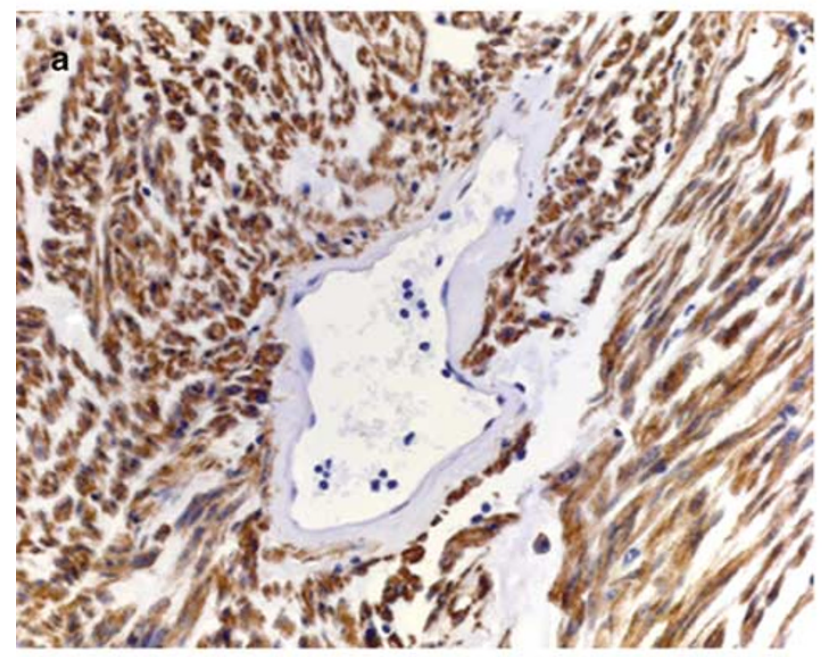

PNST: L1+ (large vessel L1-)

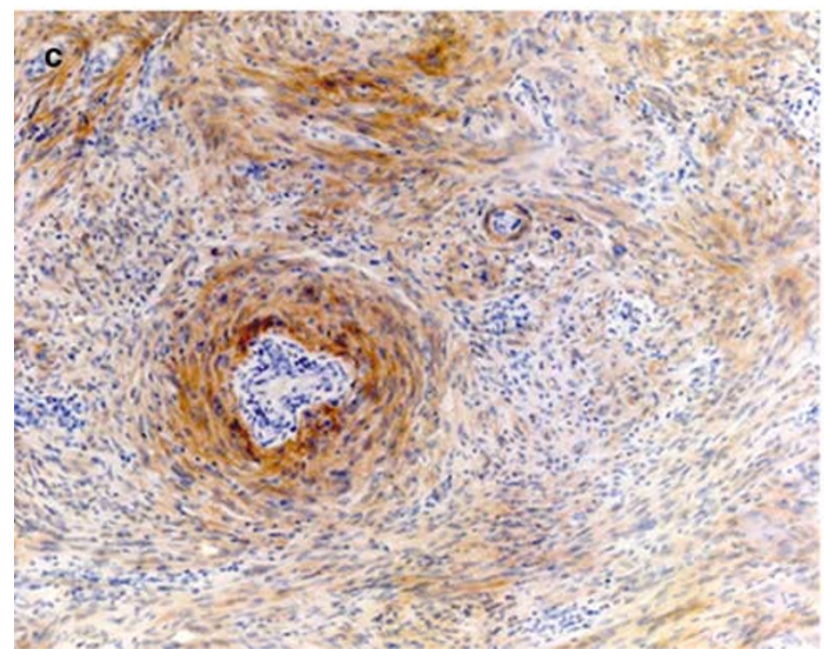

Leiomyoma: L1- (vessel L1+)

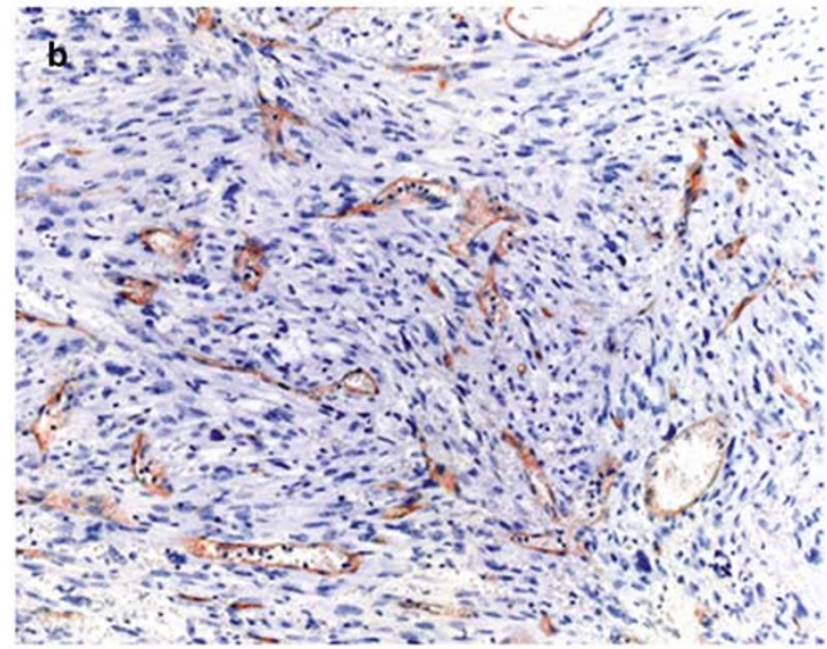

PNST: L1- (small vessel L1+)

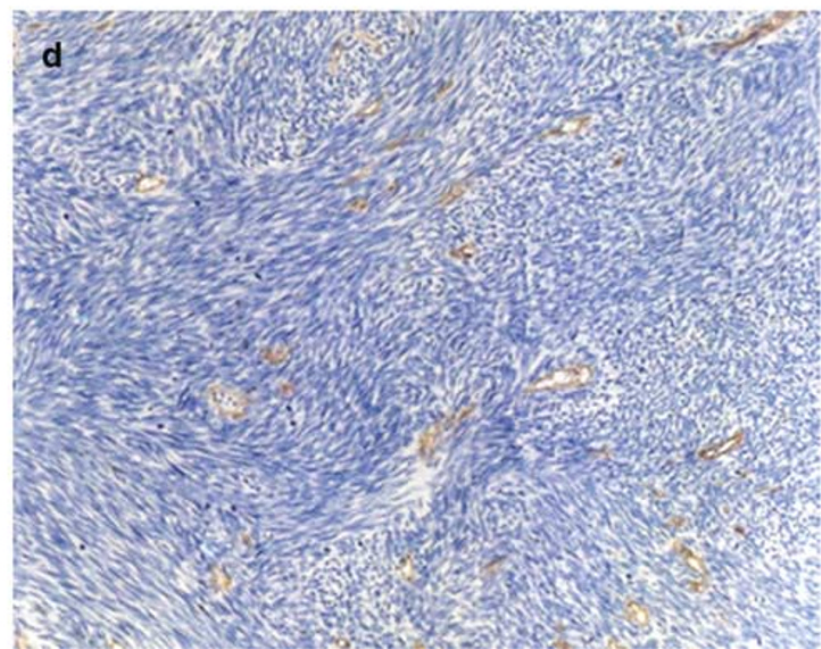

Figure 2 Differential L1 expression in vascular endothelial cells of intratumoral vessels of GISTs, leiomyomas and PNSTs. In GIST, no expression of $L 1$ was detected in intratumoral vessels (a). In contrast, $L 1$ was expressed in vascular endothelial cells of smooth muscle tumors (b) that were all L1-negative. Concerning PNSTs, L1 was detected in vascular endothelial cells of large (c) but not of small vessels (d). (Magnification: a, b: $\times 200 ; \mathbf{c}, \mathbf{d}: \times 50$ )

malignancies, we determined $L 1$ expression in $74 \%$ of GISTs but in no smooth muscle tumor or desmoidtype fibromatosis by immunohistochemical analysis. L1 expression was also detected on interstial cells of Cajal believed to be the progenitor cells of GISTs. A trend towards worse outcome of L1-positive patients with no L1-negative patient dying due to GIST was determined, which might turn out to be significant with larger patient numbers and longer follow-up time. Furthermore, four of 11 patients with tumor recurrence within the L1-positive arm with worse survival receive Imatinib and all are still alive, and since this therapy is known to improve outcome Imatinib might rather hide a stronger prognostic impact of $L 1$ expression on survival of GIST patients in the present study. ${ }^{4}$
Although $c$-kit expression is the established standard in the identification of GISTs, additional prognostic markers are required. Cell adhesion molecules have an essential function in tumor progression, and the cell adhesion molecule L1 (CD171) has been paradigmatic. ${ }^{19}$ Studies using DNA microarray technique have revealed a distinct gene expression profile in GISTs allowing distinguishing these tumors from mesenchymal malignancies. ${ }^{29-31}$ While high-quality antibodies are often lacking for the genes identified in these studies, L1 can easily be analyzed by immunohistochemistry on formalin-fixed tissues. Expression of malignancy-associated markers, like for instance $H I F-1 \alpha$, STAT3 or VEGF, has been shown in GISTs. ${ }^{32-34}$ 

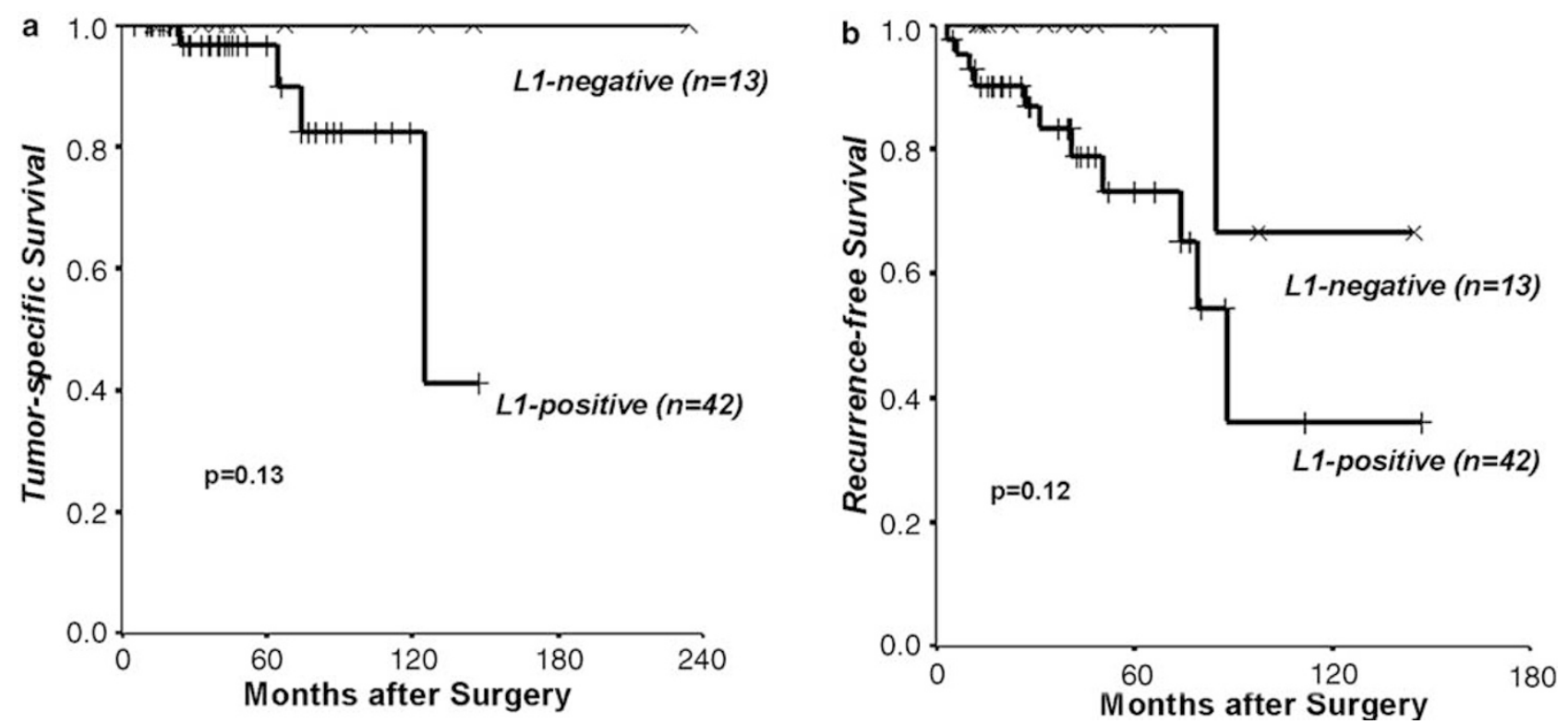

Figure 3 Kaplan-Meier curve for tumor-specific (a) and recurrence-free (b) survival and L1 expression in GISTs. Patients that did not present with metastases (M0) at initial diagnosis and whose primary tumors were surgically completely resected (R0) were included for survival analysis $(n=55)$. $P$-values were calculated with log-rank test.

The observations of this study are in agreement with previous studies showing expression of $L 1$ in different tumors of neuroectodermal origin, such as melanomas, neuroendocrine tumors of the pancreas and skin. ${ }^{2,35,36}$ In accordance with our finding, expression of $L 1$ in PNSTs by microarray expression profiling was reported recently. ${ }^{37}$ L1 was also described in non-neuroectodermal tumors like uterine, ovarian or colon carcinomas. ${ }^{28,38}$ In some of these studies a correlation with tumor-specific survival was found and the survival data of the present study might be a hint towards a similar finding in GISTs. GISTs have also been shown to express other proteins of the nervous system, such as glial fibrillary acidic protein (GFAP) and neurofilament. ${ }^{39}$ The present study shows that a neuronal adhesion molecule like L1 can also be highly expressed in GISTs. A metalloproteinase (ADAM10) cleaves $L 1$ from the tumor cell surface and soluble $L 1$ was detected in serum of tumor patients proposing an additional diagnostic tool in GIST patients. ${ }^{28}$

A differential expression pattern on tumor vessels was found as a bystander result in this study. $L 1$ was not detected in vascular endothelial cells in tumor vessels of GISTs, but on small vessels of PNSTs and small and large vessels of L1-negative leiomyomas and leiomyosarcoma. Given that tumor cells expressing $L 1$ can interact with multiple vascular or platelet integrins, $L 1$ up-regulation could play an important role in extravasation of tumor cells during metastatic spread. ${ }^{25}$ Expression of $L 1$ was also described in tumor vessels in renal cell carcinomas. ${ }^{27}$ The present study represents a similar finding, however, the reason for the vascular L1 expression in smooth muscle tumors and PNSTs but not in GISTs remains unclear. Further examinations have to elucidate a possible role of $L 1$ in tumor angiogenesis in different tumor entities.

In summary, our results indicate that $L 1$ is highly expressed in GISTs and at least hint towards a negative impact on survival and recurrence. L1 could serve as a tumor marker in differential diagnosis with regard to other soft tissue tumors in general, but particularly pertinent to smooth muscle tumors and desmoid-type fibromatosis of the gastrointestinal tract. Future studies might focus on detection of $L 1$ in the serum of GIST patients and further examine the impact of $L 1$ expression on survival.

\section{Acknowledgements}

We thank Petra Schroeder for excellent technical assistance. Financial support for this study was provided by research grants from the Hamburger Krebsgesellschaft e. V. (JTK, EFY, JRI).

\section{References}

1 Fletcher CD, Berman JJ, Corless C, et al. Diagnosis of gastrointestinal stromal tumors: a consensus approach. Int J Surg Pathol 2002;10:81-89.

2 DeMatteo RP, Lewis JJ, Leung D, et al. Two hundred gastrointestinal stromal tumors: recurrence patterns and prognostic factors for survival. Ann Surg 2000; 231:51-58

3 Miettinen M, El-Rifai W, Sobin L HL, et al. Evaluation of malignancy and prognosis of gastrointestinal stromal tumors: a review. Hum Pathol 2002;33:478-483. 
4 Demetri GD, von Mehren M, Blanke CD, et al. Efficacy and safety of imatinib mesylate in advanced gastrointestinal stromal tumors. N Engl J Med 2002;347: 472-480.

5 Miettinen M, Majidi M, Lasota J. Pathology and diagnostic criteria of gastrointestinal stromal tumors (GISTs): a review. Eur J Cancer 2002;38(Suppl 5):S39-51.

6 Miettinen M, Sarlomo-Rikala M, Sobin LH, et al. Esophageal stromal tumors: a clinicopathologic, immunohistochemical, and molecular genetic study of 17 cases and comparison with esophageal leiomyomas and leiomyosarcomas. Am J Surg Pathol 2000;24:211-222.

7 Miettinen M, Sarlomo-Rikala M, Sobin LH. Mesenchymal tumors of muscularis mucosae of colon and rectum are benign leiomyomas that should be separated from gastrointestinal stromal tumors-a clinicopathologic and immunohistochemical study of eighty-eight cases. Mod Pathol 2001;14:950-956.

8 Miettinen M, Sobin LH, Lasota J. Gastrointestinal stromal tumors of the stomach: a clinicopathologic, immunohistochemical, and molecular genetic study of 1765 cases with long-term follow-up. Am J Surg Pathol 2005;29:52-68.

9 Graadt van Roggen JF, van Velthuysen ML, Hogendoorn PC. The histopathological differential diagnosis of gastrointestinal stromal tumours. J Clin Pathol 2001; 54:96-102.

10 Ohnishi T, Matsumura H, Izumoto S, et al. A novel model of glioma cell invasion using organotypic brain slice culture. Cancer Res 1998;58:2935-2940.

11 Schachner M. Neural recognition molecules and synaptic plasticity. Curr Opin Cell Biol 1997;9:627-634.

12 Brummendorf T, Kenwrick S, Rathjen FG. Neural cell recognition molecule L1: from cell biology to human hereditary brain malformations. Curr Opin Neurobiol 1998;8:87-97.

13 Hortsch M. The L1 family of neural cell adhesion molecules: old proteins performing new tricks. Neuron 1996;17:587-593.

14 De Angelis E, MacFarlane J, Du JS, et al. Pathological missense mutations of neural cell adhesion molecule L1 affect homophilic and heterophilic binding activities. EMBO J 1999;18:4744-4753.

15 Oleszewski M, Beer S, Katich S, et al. Integrin and neurocan binding to L1 involves distinct Ig domains. J Biol Chem 1999;274:24602-24610.

16 Fogel M, Mechtersheimer S, Huszar M, et al. L1 adhesion molecule (CD 171) in development and progression of human malignant melanoma. Cancer Lett 2003;189:237-247.

17 Gil OD, Sakurai T, Bradley AE, et al. Ankyrin binding mediates L1CAM interactions with static components of the cytoskeleton and inhibits retrograde movement of L1CAM on the cell surface. J Cell Biol 2003;162: 719-730.

18 Gutwein $\mathrm{P}$, Mechtersheimer S, Riedle S, et al. ADAM10-mediated cleavage of L1 adhesion molecule at the cell surface and in released membrane vesicles. FASEB J 2003;17:292-294.

19 Kowitz A, Kadmon G, Verschueren H, et al. Expression of L1 cell adhesion molecule is associated with lymphoma growth and metastasis. Clin Exp Metastasis 1993;11:419-429.

20 Linnemann D, Raz A, Bock E. Differential expression of cell adhesion molecules in variants of K1735 melanoma cells differing in metastatic capacity. Int J Cancer 1989;43:709-712.
21 Miyahara R, Tanaka F, Nakagawa T, et al. Expression of neural cell adhesion molecules (polysialylated form of neural cell adhesion molecule and L1-cell adhesion molecule) on resected small cell lung cancer specimens: in relation to proliferation state. J Surg Oncol 2001;77:49-54.

22 Thies A, Schachner M, Moll I, et al. Overexpression of the cell adhesion molecule L1 is associated with metastasis in cutaneous malignant melanoma. Eur J Cancer 2002;38:1708-1716.

23 Gutwein P, Oleszewski M, Mechtersheimer S, et al. Role of Src kinases in the ADAM-mediated release of L1 adhesion molecule from human tumor cells. J Biol Chem 2000;275:15490-15497.

24 Kalus I, Schnegelsberg B, Seidah NG, et al. The proprotein convertase PC5A and a metalloprotease are involved in the proteolytic processing of the neural adhesion molecule L1. J Biol Chem 2003;278: 10381-10388.

25 Mechtersheimer S, Gutwein P, Agmon-Levin N, et al. Ectodomain shedding of L1 adhesion molecule promotes cell migration by autocrine binding to integrins. J Cell Biol 2001;155:661-673.

26 Montgomery AM, Becker JC, Siu CH, et al. Human neural cell adhesion molecule L1 and rat homologue NILE are ligands for integrin alpha v beta 3. J Cell Biol 1996;132:475-485.

27 Allory Y, Matsuoka Y, Bazille C, et al. The L1 cell adhesion molecule is induced in renal cancer cells and correlates with metastasis in clear cell carcinomas. Clin Cancer Res 2005;11:1190-1197.

28 Fogel M, Gutwein P, Mechtersheimer S, et al. L1 expression as a predictor of progression and survival in patients with uterine and ovarian carcinomas. Lancet 2003;362:869-875.

29 Allander SV, Nupponen NN, Ringner $\mathrm{M}$, et al. Gastrointestinal stromal tumors with KIT mutations exhibit a remarkably homogeneous gene expression profile. Cancer Res 2001;61:8624-8628.

30 Blay P, Astudillo A, Buesa JM, et al. Protein kinase C theta is highly expressed in gastrointestinal stromal tumors but not in other mesenchymal neoplasias. Clin Cancer Res 2004;10(Part 1):4089-4095.

31 Nielsen TO, West RB, Linn SC, et al. Molecular characterisation of soft tissue tumours: a gene expression study. Lancet 2002;359:1301-1307.

32 Paner GP, Silberman S, Hartman G, et al. Analysis of signal transducer and activator of transcription 3 (STAT3) in gastrointestinal stromal tumors. Anticancer Res 2003;23:2253-2260.

33 Takahashi R, Tanaka S, Hiyama T, et al. Hypoxiainducible factor-1alpha expression and angiogenesis in gastrointestinal stromal tumor of the stomach. Oncol Rep 2003;10:797-802.

34 Takahashi R, Tanaka S, Kitadai Y, et al. Expression of vascular endothelial growth factor and angiogenesis in gastrointestinal stromal tumor of the stomach. Oncology 2003;64:266-274.

35 Deichmann M, Kurzen H, Egner U, et al. Adhesion molecules CD171 (L1CAM) and CD24 are expressed by primary neuroendocrine carcinomas of the skin (Merkel cell carcinomas). J Cutan Pathol 2003;30: 363-368.

36 Kaifi JT, Zinnkann U, Yekebas EF, et al. L1 cell adhesion molecule (CD171) as a potential marker for poorly differentiated pancreatic neuroendocrine carcinomas. World J Gastroenterol 2006;12:95-99. 
37 Watson MA, Perry A, Tihan T, et al. Gene expression profiling reveals unique molecular subtypes of Neurofibromatosis type I-associated and sporadic malignant peripheral nerve sheath tumors. Brain Pathol 2004; 14:297-303.

38 Gavert N, Conacci-Sorrell M, Gast D, et al. L1, a novel target of beta-catenin signaling, transforms cells and is expressed at the invasive front of colon cancers. J Cell Biol 2005;168:633-642.

39 Sarlomo-Rikala M, Tsujimura T, Lendahl U, et al. Patterns of nestin and other intermediate filament expression distinguish between gastrointestinal stromal tumors, leiomyomas and schwannomas. Apmis 2002;110:499-507. 